\title{
Comparative studies on Orobanche cernua L. and $O$. cumana Wallr. (Orobanchaceae) in the Iberian Peninsula
}

\author{
ANTONIO J. PUJADAS-SALVÀ* \\ Departamento de Ciencias y Recursos Agrícolas y Forestales, Universidad de Córdoba, \\ Apartado 3048, E-14080 Córdoba, Spain
}

LEONARDO VELASCO

Instituto de Agricultura Sostenible (CSIC), Apartado 4084, E-14080 Córdoba, Spain

Received Fuly 1999; accepted for publication March 2000

\begin{abstract}
The systematic treatment of Orobanche cumana Wallr. and O. cernua $\mathrm{L}$. is controversial. Whereas some authors consider $O$. cumana as an infraspecific taxon of $O$. cernua, others treat it as a separate species. Furthermore, the nomenclature of the sunflower broomrape is unclear, and both names are found without qualification in the literature. The objective of the present study has been to evaluate the distribution, morphology, and ecology of $O$. cernua and $O$. cumana in the Iberian Peninsula, as well as their seed oil fatty acid profile, which is a trait of great chemotaxonomic value. O. сеrnua was found parasitizing wild Compositae, mainly Artemisia spp., whereas $O$. cumana was exclusively found on cultivated sunflower. Both species clearly differ in morphological traits, especially plant height and build, length and structure of the inflorescence, corolla length and colour. Both species are characterized by contrasting seed oil fatty acid profiles, with high oleic acid concentration in O. cernua and high linoleic acid concentration in O. cumana. The evaluation of both taxa in the Iberian Peninsula gives additional support to those authors that treat them as separate species.
\end{abstract}

(C) 2000 The Linnean Society of London

ADDITIONAL KEY WORDS:- broomrape - fatty acids - parasitic plants - seed oil sunflower - taxonomy.

\section{CONTENTS}

Introduction . . . . . . . . . . . . . . . . . . . . . 514

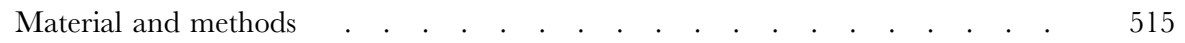

Material studied . . . . . . . . . . . . . . 515

Analysis of seed oil content and fatty acid composition . . . . . . . . 519

Results and discussion . . . . . . . . . . . . . . . . . . . 519

Morphological description . . . . . . . . . . . . . . . . . 519

* Corresponding author. E-mail: crlpusaa@uco.es 
Habitat, distribution and phenology . . . . . . . . . . . . . 523

Oil content and fatty acid composition . . . . . . . . . . . . . . . 523

Conclusions . . . . . . . . . . . . . . . . . . . . . . . 525

Acknowledgements . . . . . . . . . . . . . . . . . . . . . . . . 525

References . . . . . . . . . . . . . . . . . . . . . 526

\section{INTRODUCTION}

Orobanche cernua L. was described by Carl Linnaeus, on materials collected by Pehr Löfling (Loefling) near Aranjuez, central Spain (Loefling, 1758). It parasitizes different species of the Compositae, being most frequently found on plants of the genus Artemisia. The main distribution area for this species is the Mediterranean region to central Asia (Reuter, 1847; Beck-Mannagetta, 1930). Following the International Code of Botanical Nomenclature (Greuter, 1988) we use the authority O. cernua L. instead of the more traditional $O$. cernua Loefl., as proposed by López-González (1990).

The description of O. cumana was made by Wallroth (1825), on plants collected in desert areas of south-western Asia and south-eastern Europe. Beck-Mannagetta (1930), who considered this species as a variety of O. cernua, reported central Asia to south-eastern Europe as the main distribution area for $O$. cumana. Originally, $O$. cumana parasitized Artemisia spp. exclusively (Venkov \& Bozoukov, 1994). After the introduction of the sunflower (Helianthus annuus L.) cultivation in Europe, plants of $O$. cumana were detected for the first time parasitizing this crop in north-eastern Bulgaria in 1935 (Encheneva \& Shindrova, 1994). In the Iberian Peninsula, it was first described parasitizing cultivated sunflower in 1958 (Díaz-Celayeta, 1974). Since then, O. cumana has spread over the whole sunflower cultivation area (c. 1 million ha in central- and southern Spain) representing currently a serious problem limiting sunflower productivity (Alonso et al., 1996).

Orobanche cernua L. and O. cumana Wallr. have been traditionally considered as very closely related taxa. Thus, $O$. cumana has been recognized as an infraspecific taxon of O. сеrnua (Beck-Mannagetta, 1890, 1930; Rechinger, 1943; Greuter, Burdet \& Long, 1989), or both names have been used as synonymous by some authors (Bonnier, 1926; Chater \& Webb, 1972; Bischof, 1978; Pignatti, 1982). Other authors, however, have classified both taxa as separate species (Reuter, 1847; Jackson, 1895; Joel, 1987; Uhlich, Pusch \& Barthel, 1995; Kreutz, 1995). In parallel with the lack of agreement among botanists, some plant pathologists refer to sunflower broomrape as O. cernua (e.g. Díaz-Gelayeta, 1974; González-Torres, Jiménez-Díaz \& MeleroVara, 1982; Castejón-Muñoz, Romero-Muñoz \& García-Torres, 1991; Parker, 1994; Alonso et al., 1996; Sukno, Melero-Vara \& Fernández-Martínez, 1999), whereas others use O. cumana (e.g. Petrov, 1968; Cherzhentseava, 1978; Vrânceanu et al., 1986; Joel, 1988; Abu-Irmaileh, 1994; Antonova \& Ter-Borg, 1996).

Despite the mentioned discrepancies, recent molecular studies clearly support the separation of O. cernua and O. cumana into different species (Katzir et al., 1996; Paran, Gidoni \& Jacobsohn, 1997; Joel, Portnoy \& Katzir, 1998; Pujadas \& Thalouarn, 1998).

The fatty acid profile of the seed oil has great systematic value in the plant kingdom (Gibbs, 1974), and there are many studies reporting phylogenetic relationships paralleled by differences in the fatty profile of the seed oil (e.g. Velasco \& Goffman, 1999; Aitzetmüller, Tsevegüren \& Werner, 1999). This trait has not yet been evaluated in the Orobanchaceae. 
In this paper we report a comparative evaluation of $O$. cernua and $O$. cumana in the Iberian Peninsula, describing their morphology, distribution, habitat, phenology, and seed oil content and fatty acid composition.

\section{MATERIAL AND METHODS}

\section{Material studied}

Unless otherwise cited, plant material of $O$. cernua and $O$. cumana was collected by the first author. Voucher specimens are deposited in the Herbarium of the University of Córdoba (COA), Spain. Furthermore, the following herbaria have been consulted: ABH, ALME, BC, BCC, BCF, COA, C ${ }^{\circ}$ LA SALLE DE ALMERIA, GDA, GDAC, JACA, JAEN, HBIL, MA, MACB, MAF, MGC, MUB, SEV, VAB \& VAL.

Orobanche cernua L., Iter Hisp. 152 (1758)

SPAIN. Aligante: Confrides, Sierra Aitana, pr. Font de l'Abre, 1200 m, YH3582, 18.v.1992, 7.L. Solanas, De La Torre \& Crespo (ABH 14832). Dehesa de Campoamor, Orihuela, XH93O4, $100 \mathrm{~m}, 31 . v .1996$, S. Espinar et al. (ABH 31825). Serra del Portitxol, on Artemisia campestris, 2.iv.1958, A. et O. de Bolòs (BC 261398). Sierra de Orihuela, 30SXH71, 15.iv.1984, T. Molero \& A. Rovira (BCF 39860). Almeria: La Hoya, 16.iii.1958, Hno. M. Mauricio \& R. Sagredo (ALME 1721). Serón, Las Menas, on Artemisia sp., $1300 \mathrm{~m}, 11$. viii.1958, Hno. M. Mauricio \& R. Sagredo (ALME 1718). Tabernas, Venta de los Yesos, growing on Artemisia in wheatfield, 30SWG60, 520-550 m, 3.vi.1967, P. W. Ball, A. O. Chater, I. K. Ferguson \& B. Valdés (ALME 1705 and SEV 4861). NW Almería, Barranco de Cerro Caguela, N side of Rambla de Belén, stony slopes, limestone, 30SWG47, 100-300 m, 6.vi.1967, P. W. Ball, A. O. Chater, I. K. Ferguson \& B. Valdés (ALME 1706 and SEV 4860). Between Tabernas and Gérgal, Yesoncillo de Enmedio, on gypsaceous soils, 30SWG40, 500-600 m, 30.iv.1983, R. Lázaro Suau (ALME s/n). Near Los Cuernos, 30SWG6465, 1500 m, 8.vi.1988, M. Cueto (ALME s/n). Tabernas, on Artemisia barrelieri, 30SWF4898, $400 \mathrm{~m}$, 29.iv.1989, A. Pujadas \& P. Poyato (COA 13632). Níjar, 30SWF7191, 17.iii.1990, A. Pujadas et al. (COA 13464). Salinas Cabo de Gata, 30SWF7069, 3 m, 17.iii.1990, A. Pujadas et al. (COA 17366). Cabo de Gata, Cerro Vela Blanca, 30SWF7364, 20 m, 17.iii.1990, A. Pujadas et al. (COA 17368). Los Genoveses, Cabo de Gata, 30SWF3976, 250 m, 17.iii.1990, A. Pujadas et al. (COA 17369). San José, 30SWF7968, 10.vii.1990, A. Pujadas (COA 17358). Yesera de Gafarillos, Sorbas, 30SWF8702, 18.iv.1992, E. Hernández \& P. Contreras (COA 17365). Barriada de Alcora, 30SWF2391, $760 \mathrm{~m}$, 13.v.1993, A. Pujadas, A. Lora \& M. Mazariegos (COA 17359). Cerro del Buho, Tabernas, on Artemisia barrelieri, 30SWG5202, 14.v.1993, A. Pujadas, A. Lora \& M. Mazariegos (COA 17360). Cerro Santa Fe, on Artemisia barrelieri, 30SWF7670, $350 \mathrm{~m}$, 23.iii.1994, A. Pujadas (COA 17355). Enix, on Artemisia barrelieri, 30SWF3879, 7.iv.1994, A. Pujadas (COA 17363). Cerro Majada Redonda, on Launaea lanifera, 30SWF8174, 300 m, 13.iv.1994, A. Pujadas (COA 17353). N del Barranco del Sabinar, cortijo El Rincón de Martos, 30SWF7470, 300 m, 19.iv.1994, A. Pujadas (COA 17356). Barranco El Palmer, 30SWF3976, $250 \mathrm{~m}$, 22.iv.1994, A. Pujadas (COA 17370). Cerro El Fraile, on Artemisia barrelieri, 30SWF8271, 40 m, 2.v.1994, A. Pujadas (COA 17371). El Garbanzal, on Artemisia barrelieri, 30SWF7874, $200 \mathrm{~m}$, 
6.v.1994, A. Pujadas (COA 17352). Las Pocicas, on Artemisia glutinosa, 30SWG7548, 700 m, 11.v.1994, A. Pujadas (COA 17349). Arroyo del Saliente, Los Cerricos, Oria, 30SWG7254, 1000 m, 11.v.1994, A. Pujadas (COA 17372). Santuario El Saliente, on Artemisia glutinosa, 30SWG7453, $900 \mathrm{~m}, 16 . v .1994$, A. Pujadas (COA 17350). Barranco del Tartel, Sierra de Gádor, on Artemisia barrelieri, 500 m, 22.v.1994, A. Pujadas (COA 17351). Cerro de Mónsul, 30SWF7667, 200 m, 1.vi.1994, A. Pujadas (COA 17357). Tabernas, Venta de los Yesos, 30SWG6204, 18.v.1996, A.Pujadas (COA 20595). Sierra Nevada, Alhabia, on Launaea lanifera Pau, 430 m, 5.iv.1997, f. Pusch (COA 23584). Gueva de los Ubedas, 30SWF6385, 16.vi.1998, L. Plaza (COA 25549). Palacio de Arboleas, 23.iv.1982, P. Sánchez (GDAC 14360). Adra to La Parra, 17.iv.1990, M. 7. Martínez \& L. Gutiérrez (GDAC 35199). Las Menas, Serón, on Artemisia sp., $1300 \mathrm{~m}, \mathrm{~s} / \mathrm{f}, R$. Sagredo (H $\mathrm{H}^{\circ}$ LA SALLE s/n). Between Rioja and Tabernas, 19.iv.1967, $R$. Sagredo (H $\mathrm{H}^{\circ}$ LA SALLE s/n). Venta de los Yesos, $2 \mathrm{~km}$ from Uleila, 19.iv.1967, R. Sagredo (H' LA SALLE s/n). El Fonte, Cortijo, 19.iv.1967,

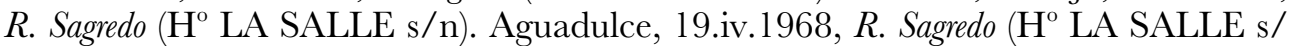
n). Faro Cabo de Gata, 3.v.1968, R. Sagredo (H' LA SALLE s/n). Los Castaños, 14.iv.1973, R. Sagredo (H $\mathrm{H}^{\circ}$ LA SALLE s/n). Serrata, 20.iv.1973, R. Sagredo $\left(\mathrm{H}^{\circ}\right.$ LA SALLE s/n). Tabernas, near Rambla de Tabernas, 30SWG60, 450 m, 2.iii.1955, P. Montserrat (JACA 5255). Carboneras, Presa de Agua Amarga, 10 m, 12.v.1982, A. Charpin \& C. Defferrard (MA 294125). Cabo de Gata, 10.vi.1985, M. S. Sanz, A. Nebot \&̊ F. Boisset (VAB 904408). Cáceres: Hervás, on Artemisia glutinosa, vi, M. Rivas Mateos (MAF 36157). Castellón: Segorbe, on Artemisia herba-alba var. valentina, 19.v.1928, C. Pau (BC 822104, MA 114871 and MAF 36156). Segorbe, Monte Castelli, on Artemisia valentina Lam., 1880, C. Pau (MA 114873). Segorbe, on Artemisia valentina, 1918, C. Pau (MA 114872). Granada: Castell de Ferro, Cala Rijana, on Launaea lanifera Pau, 28.ii.1995, A. Pujadas \& P. Poyato (COA 17627). Monachil, Base del Trevenque, Cortijo de la Fuente del Hervidero, on Artemisia campestris subsp. glutinosa, 30SVG5304, $1360 \mathrm{~m}, 13 . v i i .1997$, A. Pujadas (COA 23548). Cuenca del Benimar, Ugíjar, VF9489, 1.v.1984, f. Guirado (GDAC 28833). Lanjarón, La Alpujarra, 1902, C. Pau (MA 114870). Between El Marquesado and Alquife, hillsides with pines, on shales, VG 9011, 1700 m, 16.vi.1988, B. Valdés et al. (SEV s/n). Huesca: Serreta Negra de Fraga, 14.v.1977, Martín \& Molero (BCF 39859). Fraga, 31TBF7399, 330 m, 31.v.1960, P. Montserrat (JACA 14160). Candasnos, La Valcuerna, 31TBF5094, 350 m, 5.v.1971, P. Montserrat (JACA 9247 1). Sariñena, Laguna, 30TYM3430, $280 \mathrm{~m}$, 3.vi.1980, P. \& G. Montserrat (JACA 121580). Ballerías, 30TYM34, $370 \mathrm{~m}, 22 . v .1988, G$. \& F. Montserrat (JACA 88888). Monzón, 31TBG6742, $360 \mathrm{~m}, 13 . v .1988$, f. A. Sesé (JACA 607088). Alcolea de Cinca, La Codera, on Artemisia herba-alba, 31TBG6219, 220 m, 14.iv.1995, f. V. Ferrández (JACA 515595). Albalatillo, 30TYM3619, $260 \mathrm{~m}, 12 . v i .1995$, L. Villar, 7. A. Sesé \& C. Pedrocchi (JACA s/n).JAén: Cabra del Santo Cristo, Base de las Atarillas, 30SVG7377, 10.v.1996, A. Pujadas \& A. Lora (COA 22113). Jódar, Chozón del Lobo, on Artemisia barrelieri Besser, 30SVG7578, 10.v.1996, A. Pujadas \& A. Lora (COA 22121). Cabra del Santo Cristo, Las Ramblas, on Artemisia barrelieri Besser, 30SVG7477, 14.vi.1996, A. Pujadas (COA 20592). Cabra del Santo Cristo, Las Jarosas, on Artemisia barrelieri Besser, 30SVG7681, 14.vi.1996, A. Pujadas (COA 20593). Bélmez de Moraleda, base of Sierra de la Cruz, on Artemisia barrelieri Besser, 30SVG6978, 760 m, 14.vi.1996, A. Pujadas, COA 20594. Cabra del Santo Cristo, Loma del Campillo, VG-78, 800 m, 11.v.1979, G. Fernández López (JAEN 79463). La Rioja: Briones, vi.1939, s/l. (BCF 39892). Logroño to Fuenmayor, on Artemisia herba-alba, v.1920, Zubia (MA 114865). 
LÉRIDA: Segrià, near Alcarrás, on Artemisia herba-alba, 11.vi.1962, F. Masclans (BC 597186). Tossal de Prop de Miralcamp, CG20, 10.vi.1987, A. Mayoral (HBIL 7144). Madrid: Aranjuez, on Artemisia valentina, 25.v.1919, C. Vicioso (BC 46699). Valdemoro, v.1915, C. Vicioso (MA 114861). Murcia: Cabo de Palos, Cala Reona, 30SYG0267, on Artemisia barrelieri Besser, 18.v.1996, A. Pujadas (COA 22114). Lorca, on Artemisia spp., -s/f-, Cánovas (GDA s/n). Sierra de Columbares, XG7399, 400 m, 14.iii.1980, F. Alcaraz (MUB 2885). Base of Pico Mirabete, 30.v.1985, 7. M. Estremera (MUB 24335). Lorca, XG1578, 450 m, 1.v.1996, A. Robledo-Miras (MUB 17220). Cehegín, Sierra de la Puerta, 30SXH 0826, 550 m, 3.v.1986, C. Selma (MUB 28633). Jumilla, El Cabezo, 27.iv.1991, A. I. Fiménez-Piqueras (MUB 39770). Palencia: Castillo de Magaz, 30TUM8049, 24.vii.1988, C. López छ̈ A. Romero-Abelló (MACB 50326). TARragona: Delta de L'Ebre, Cap a L'Aufacada, CI10, on dunes near the sea, 16.v.1983, C. Benedí \&̊ 7. Molero (BCF 39409). Teruel: Tornos, Gallocanta, 30TXL2835, $1000 \mathrm{~m}$, on Artemisia cf. gallica, 5.vii.1972, P. Montserrat \& L. Villar (JACA 438372). Las Parras de Martín, Soto de la Solana, on Artemisia sp., 22.vi.1881, Badal (MA 114868). Toledo: Ontígola, 30SVK4829, 600 m, 15.vi.1959, P. Montserrat (JACA 35559). Seseña, Dehesa Nueva del Rey, 30TVK4332, on Artemisia barrelieri, 23.v.1998, A. Pujadas (COA 24660). Valencia: Font del Plà, Ermita de Santa Bàrbara, Bocairent, 30SYH09, 800 m, on Artemisia campestris, 21.v.1988, F. R. Nebot (VAB 901747). ZARAGOZA: Chiprana, La Laguneta Salada, on Artemisia herba-alba, 21.v.1954, O. de Bolós \& F. Br.-Blq. (BC 128440). Laguna de Pito, Monegros, 23.v.1988, A. Carrillo Eீ 7. M. Ninot (BCG s/n). Los Monegros, Osera-Monegrillo, 30TYM0102, $250 \mathrm{~m}$, 1.vi.1956, P. Montserrat (JACA 9856). Sástago, Monte de Rueda, 30TYL2376, 180 m, 12.v.1959, P. Montserrat (JACA 21659). Bárdenas Reales, c. Tres Mugas, 30TXM3283, 16.v.1988, D. Gómez (JACA 60988). Mequinenza, Val de Beauradó, gypsaceous soil, on Artemisia herba-alba, 31TBF7586, $130 \mathrm{~m}, \mathcal{F}$. V. Fernández (JACA 517895). Daroca, 27.vi.1909, C. Vicioso (MA 114867). Calatayud, Campiel, 7.vi.1910, C. Vicioso (MA 114866). Valdehurón, Calatayud, 28.v.1988, f. A. U. (VAB 940396).

Orobanche cumana Wallr., Orob. Gen. Diask. 58 (1825)

CÁdiz: Puerto Serrano, on cultivated sunflower (Helianthus annuus L.), 3.vi.1989, 7. Oliveira (COA 13524). Jerez de la Frontera, .vii.1995, 7. Fernández Escobar, CA195 (COA 28285). Córdoba: Córdoba, Finca Ruano, on cultivated sunflower, 9.vi.1993, F. Cartujo (COA 17472). Córdoba, Parque Cruz Conde, 16.vi.1994, P. Blanco (COA 17471). Córdoba, near Airport, on cultivated sunflower, 30.vi.1996, A. Lora (COA 22078). Córdoba, Huerta de San Luis, on cultivated sunflower, 30SVG4092, 2.vii.1996, A. Lora \& A. Pujadas (COA 22077). Córdoba, Cortijo del Pardito, on cultivated sunflower, 30SUG4377, 16.vii.1996, A. Moyano \& A. Pujadas (COA 22115). Puente Genil, .vii.1994, F. Alvarado, CO194 (COA 28286). Córdoba, .vii.1996, f. M. Melero, CO196 (COA 28287). Cuenca: Villarejo de Penesteban, .viii.1992, f. M. Melero, GU192 (COA 28288). Alcázar del Rey, .ix.1994, f. M. Melero, CU494, (COA 28289). Km. 17,800 of Cuenca road, 2.viii. 1978, M. Velayos (MACB 47083). Granada: Atarfe, on cultivated sunflower (oilseed), 14.ix.1995, A. Pujadas (COA 17629). Málaga: Antequera, on cultivated sunflower (oilseed), 3.vi.1989, f. Oiveira (COA 13569). Campillos, on cultivated sunflower (confectionery), 13.vi.1989, f. Oliveira (COA 13525). Between Campillos and Antequera, $20 \mathrm{~km}$, on cultivated sunflower (oilseed), 13.vi.1989, f. Oliveira (COA 13568). Near Laguna de Fuente de Piedra, 
TABLE 1. Analysed populations for seed oil content and fatty acid composition of Orobanche cernua and O. cumana from the Iberian Peninsula

\begin{tabular}{|c|c|c|}
\hline Species & Province & Reference \\
\hline O. cernua & Almería & COA 13632 \\
\hline O. cernua & Almería & COA 13464 \\
\hline O. cernua & Almería & COA 17358 \\
\hline O. cernua & Almería & COA 17365 \\
\hline O. cernua & Almería & COA 17359 \\
\hline O. cernua & Almería & COA 17360 \\
\hline O. cernua & Almería & COA 17355 \\
\hline O. cernua & Almería & COA 17363 \\
\hline O. cernua & Almería & COA 17353 \\
\hline O. cernua & Almería & COA 17356 \\
\hline O. cernua & Almería & COA 17370 \\
\hline O. cernua & Almería & COA 17352 \\
\hline O. cernua & Almería & COA 17349 \\
\hline O. cernua & Almería & COA 17372 \\
\hline O. cermua & Almería & COA 17350 \\
\hline O. cernua & Almería & COA 17351 \\
\hline O. cernua & Almería & COA 20595 \\
\hline O. cernua & Almería & COA 23584 \\
\hline O. cernua & Granada & COA 17627 \\
\hline O. cernua & Granada & COA 23548 \\
\hline O. cernua & Jaén & COA 22113 \\
\hline O. cernua & Jaén & COA 22121 \\
\hline O. cernua & Jaén & COA 20592 \\
\hline O. cernua & Jaén & COA 20593 \\
\hline O. cernua & Jaén & COA 20594 \\
\hline O. cermua & Murcia & COA 22114 \\
\hline O. cernua & Toledo & COA 24660 \\
\hline O. cumana & Cádiz & COA 28285 \\
\hline O. cumana & Córdoba & COA 22077 \\
\hline O. cumana & Córdoba & COA 22115 \\
\hline O. cumana & Córdoba & COA 28286 \\
\hline O. cumana & Córdoba & COA 28287 \\
\hline O. cumana & Cuenca & COA 28288 \\
\hline O. cumana & Cuenca & COA 28289 \\
\hline O. cumana & Sevilla & COA 28295 \\
\hline O. cumana & Sevilla & COA 28296 \\
\hline O. cumana & Sevilla & COA 28290 \\
\hline O. cumana & Sevilla & COA 28293 \\
\hline O. cumana & Sevilla & COA 17460 \\
\hline O. cumana & Sevilla & COA 28292 \\
\hline O. cumana & Sevilla & COA 22079 \\
\hline O. cumana & Sevilla & COA 22080 \\
\hline O. cumana & Sevilla & COA 28291 \\
\hline O. cumana & Sevilla & COA 28294 \\
\hline
\end{tabular}

on cultivated sunflower, 8.vi.1995, A. Lora \& A. Pujadas (COA 17628). Sevilla: El Coronil, on cultivated sunflower, 16.vi.1986, L. García Torres (COA 13636). Morón to El Coronil, $9 \mathrm{~km}$, on cultivated sunflower (oilseed), 2.vii.1987, f. Oliveira (COA 13624). El Coronil to Morón, $7 \mathrm{~km}$, on cultivated sunflower (oilseed), 2.vii.1987, 7. Oliveira (COA 13633). El Coronil to Utrera, $15 \mathrm{~km}$, on cultivated sunflower (confectionery), 2.vii.1987, f. Oliveira (COA 13645). Osuna, 1.vii.1988, f. Oliveira, COA 28295. El Coronil to Montellano, $10 \mathrm{~km}$, on cultivated sunflower (oilseed), 1.vii.1988, f. Oliveira (COA 13533). El Coronil to El Arahal, $12 \mathrm{~km}$, on cultivated sunflower (confectionery), 1.vii.1988, f. Oliveira (COA 13629). El Coronil to Acabalas, 
$10 \mathrm{~km}$, on cultivated sunflower (oilseed), 1.vii.1988, f. Oliveira (COA 13643). Montellano, on cultivated sunflower (confectionery), 13.vi.1989, f. Oliveira (COA 13618). Osuna, vii.1989, 7. Oliveira (COA 28296). Écija, Sotillo Gallego, vii.1993, 7. M. Melero, SE193 (COA 28290). Écija, Finca Pavía, vii.1994, 7. Fernández, SE194 (COA 28293). Écija to Herrera, 24 km, on cultivated sunflower, 18.v.1995, A. Pujadas (COA 17460). Écija, Finca Mencía, vii.1996, 7. M. Melero, SE296 (COA 28292). Écija to Estepa, $3 \mathrm{~km}$, 1.vii.1996, A. Pujadas, A. Lora \& A. Fiménez (COA 22079). Herrera to Écija, 3 km, 1.vii.1996, A. Pujadas, A. Lora \& A. fiménez (COA 22080). Aznalcázar, near Guadiamar river, on cultivated sunflower, 29SQB4232, 22.xii.1998, A. Pujadas \& M. del Río (COA 27749). Cañada del Rosal, vii.1998, 7. A. Sainz de Tejada (COA 28291). Écija, viii.1998, f. A. Sainz de Tejada (COA 28294).

The study of the geographical distribution of $O$. cumana has been complemented with information from Díaz-Celayeta (1974), Velayos-Rodríguez (1978), GonzálezTorres et al. (1982), and Castejón-Muñoz, Romero-Muñoz \& García-Torres (1989).

\section{Analysis of seed oil content and fatty acid composition}

Seed oil content and its fatty acid composition were analysed in a set of 44 accessions for which enough seed was available. It included 27 accessions of $O$. cernua and 17 accessions of $O$. cumana (Table 1). They were determined by gas-liquid chromatography (GLC) of fatty acid methyl esters with heptadecanoic acid (17:0) as internal standard. About $10 \mathrm{mg}$ seeds were placed in a $2-\mathrm{ml}$ vial. The seeds were crushed, as finely as possible with a stainless steel rod, and $0.8 \mathrm{ml}$ petrolether was added. The vials were closed with teflon covers and maintained in a waterbath at $45^{\circ} \mathrm{C}$ for $8 \mathrm{~h}$. After that the petrolether was evaporated overnight. Fatty acid methyl esters were prepared following the procedure of Garcés \& Mancha (1993) and analysed on a Perkin Elmer gas chromatograph model Autosystem (Perkin Elmer Corporation, Norwalk, CT) equipped with a 2-m long column packed with 3\% SP2310/2\% SP-2300 on Chromosorb WAW (Supelco Inc., Bellefonte, PA, USA). The oven, detector, and injector temperatures were 185,250 , and $275^{\circ} \mathrm{C}$, respectively. The carrier gas was nitrogen, at a flow of $23 \mathrm{ml} \mathrm{min}^{-1}$. Fatty acids were identified by comparison of retention times with standards. Individual fatty acids were expressed as a percentage of the total fatty acids.

\section{RESULTS AND DISCUSSION}

\section{Morphological description}

Orobanche cernua L. in Loefl., Iter. Hisp. 152 (1758).

$=O$. hispanica Boiss., Voy. Bot. Espagne 2: 476 (1839-1845).

Iconography. Figure 1; Coste (1937: 71, fig. 2825); Kreutz (1995: 78-79); Pignatti (1982: 610).

PLANT 15-32 cm tall. STEM usually thick, with a diameter of (3)5-8(9) $\mathrm{mm}$, in the middle, bulbous at the base $(9-20 \mathrm{~mm})$, glandular-pubescent, yellowish to pale violet. LEAVEs deltate below $(8-11 \times 5-8 \mathrm{~mm})$, and oval-lanceolate above $(6-11 \times 4-7 \mathrm{~mm})$. 


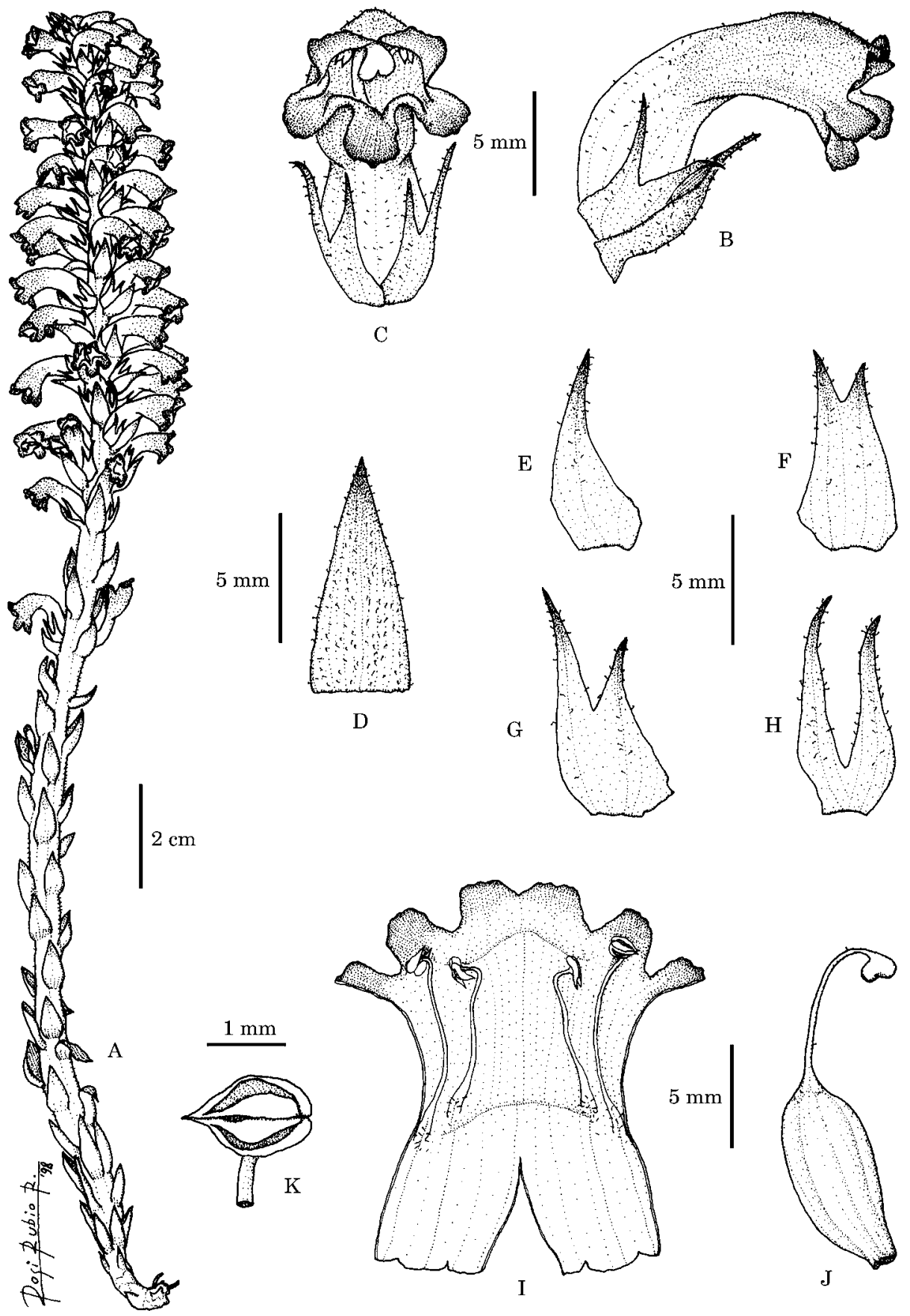

Figure 1. Orobanche cermua L., Almería, Níjar, Cerro Majada Redonda, COA 17353. A, appearance. B, flower, side view. C, flower front view. D, bract. E, F, G, H, calyx segments. I, open corolla and androecium. J, gynoecium. K, anther. 
INFLORESGENGE (4)6-17(23) $\times(1.8) 2-3(3.5) \mathrm{cm}$, dense, seldom lax at the base. BRACTS (5) 7-9(12) $\times 3.5-5 \mathrm{~mm}$, ovate to lanceolate. CALYX 6-10 mm long; calyx segments free, bifid (cleft 2.5-4 mm) to deeply bifid (cleft up to $7 \mathrm{~mm}$ ), sometimes bidentate (cleft up to $2.5 \mathrm{~mm}$ ), seldom entire, with subulate teeth. COROLLA (13)15-18 mm long, erect to erecto-patent, inflated at the base, constricted and straight in the middle, arched forward, dorsal line geniculated above the insertion of the stamens, sparsely glandular-pubescent, white at the base, blue, dark violet or purple in upper part. Upper lip of the corolla bilobed; lobes of the lower lip of the corolla 2-2.2 $\times 2.3-2.5 \mathrm{~mm}$, subequal, obovate, not ciliate. sTAMENs, adaxial with filaments 6-7 mm long, inserted obliquely (5)7-8 $\mathrm{mm}$ above the base of the corolla, glabrous or pubescent in the lower part; abaxial stamens with filaments $7-9 \mathrm{~mm}$ long, inserted (3) $4-6.5 \mathrm{~mm}$ above the base of the corolla, glabrous, glabrescent or with woolly hairs at the base. FILAMENTS without glandular hairs below the anthers. ANTHERS (1.1) 1.4-1.5 mm long, glabrous or subglabrous (few hairs up to $0.25-\mathrm{mm} l o n g$ ), white, with apiculum of $0.1-0.3 \mathrm{~mm}$. OVARY $7-11 \mathrm{~mm}$ long, glabrous or sparsely pubescent, with few glandular hairs distributed in the ventral side. STYLE with glandular hairs, sometimes subglabrous or glabrous. sTiGma bilobed, white.

Orobanche cumana Wallr., Orob. Gen. Diask. 58 (1825)

$=O$. cernua auct. non L. nec Loefl.

=O. cernua subsp. cumana (Wallr.) Soó, Feddes Repert. 83: 187 (1972).

= O. cernua var. cumana (Wallr.) Beck, Biblioth. Bot. 19: 143 (1890).

Iconography. Figure 2; Reichenbach (1829: DCXCI, fig. 926); Kreutz (1995: 90-91)

PLANT (35)40-65 cm tall. STEM slender with a diameter of (3.5)5-8(10) $\mathrm{mm}$ in the middle, clavate or slightly bulbous at the base $(9-20 \mathrm{~mm})$, glandular-pubescent, whitish. LEAVEs deltate below $(8-12 \times 6-7 \mathrm{~mm})$, and oval-lanceolate above (9-13 $\times 4-8 \mathrm{~mm})$. INFLORESCENCE, (15)22-30(38) $\times(2) 2.5-3(3.5) \mathrm{cm}$, lax, sometimes dense in the upper third. BRACTs 7-10 × 4-7 mm, ovate-lanceolate. GALYX (5)7-9 mm long; calyx segments free, entire, seldom bifid (cleft up to $0.5 \mathrm{~mm}$ ) with markedly unequal subulate teeth. Corolla (16)19-22 mm long, not or slightly inflated at the base, patent, markedly curvate, dorsal line inflected, sparsely glandular-pubescent, white at the base, white or pale blue in the upper part. Upper lip of the corolla bilobed; lobes of the lower lip of the corolla $2.2-2.5 \times 2-2.2 \mathrm{~mm}$, subequal, obovate, not ciliate. STAMENs, adaxial stamens with filaments $6-10 \mathrm{~mm}$ long, inserted 6-9 mm above the base of the corolla, glabrous, seldom glabrescent at the base; abaxial stamens with filaments 7-11(13) $\mathrm{mm}$ long, inserted (4)4.5-(6)6.5 $\mathrm{mm}$ above the base of the corolla, with a bunch of hairs at the base. FILAMENTs with sessile glandular hairs below the anthers. ANTHERs are white, (1.2)1.4-1.6 mm long, hairy (hairs up to $0.25-\mathrm{mm}$ long) at the base of the line of fusion, white, with apiculum c. $0.2 \mathrm{~mm}$. OVARY $8-10 \mathrm{~mm}$ long, glabrous. STYLE with short glandular hairs. STIGMA bilobed, white.

Orobanche cumana plants are easily recognized because they are taller, more slender, and with longer and laxer inflorescence than those of O. cernua. O. cumana also presents paler corollas (white vs pale blue) than the corollas of $O$. cernua (blue, dark violet or purple). Both species also exhibited clear differences for the corolla length and the degree of bending of its dorsal line. A comparative summary of the main morphological differences between both species is presented in Table 2 . 
522

A. J. PUJADAS-SALVÀ AND L. VELASCO
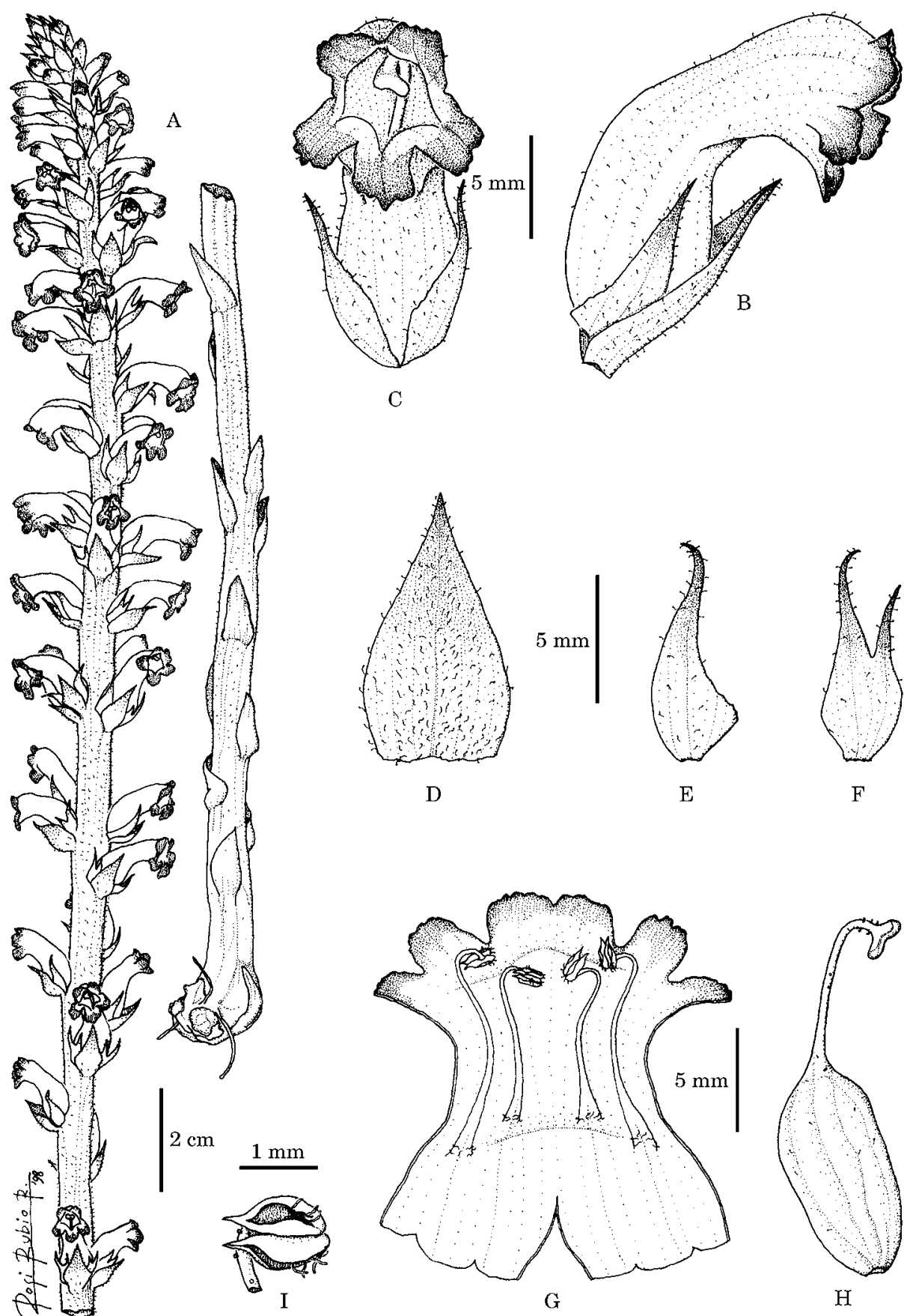

$\mathrm{D}$

$\mathrm{E}$

$\mathrm{F}$

Figure 2. Orobanche cuman Walls., Cordoba, Cortijo del Pardito, COA 23745. A, appearance. B, flower, side view. C, flower front view. D, bract. E, F, calyx segments. G, open corolla and androecium. H, gynoecium. I, anther. 
TABle 2. Morphological differences between Orobanche cernua L. and O. cumana Wallr.

\begin{tabular}{lll}
\hline Trait & O. cernua & O. cumana \\
\hline Plant $(\mathrm{cm})$ & $15-32$ & $(35) 40-65$ \\
Upper leaves $(\mathrm{mm})$ & $6-11$ & $9-13$ \\
Inflorescence $(\mathrm{cm})$ & $(4) 6-17(23)$ & $(15) 22-30(38)$ \\
Inflorescence, structure & Dense, seldom lax at the base & Lax, sometimes dense at the apex \\
Calyx (mm) & $6-10$ & $(5) 7-9$ \\
Calyx segments & Bifid to bidentate, seldom entire & Entire, sometimes bifid \\
Corolla (mm) & $(13) 15-18$ & $(16) 19-22$ \\
Corolla, position & Erect to erecto-patent & Erecto-patent to patent \\
Corolla, dorsal line & Geniculate & Inflected \\
Corolla, upper part, colour & Dark blue to violet & Whitish to pale blue \\
Filaments, apex & Glabrous & Sessile glandular hairs \\
Anthers & Glabrous or subglabrous & Hairy \\
Ovary & Glabrous or sparsely pubescent & Glabrous \\
Style & With glandular hairs to glabrous & With glandular hairs \\
Hosts & Artemisia spp., Launaea lanifera & Helianthus annuus \\
Habitat & Loamy gypsaceous soils & Alluvial loamy soils \\
Altitude (m) & 0-1500 & 0-800 \\
Anthesis & March to June & June to August \\
\hline
\end{tabular}

\section{Habitat, distribution and phenology}

Orobanche cernua was found on loamy gypsaceous soils, in arid areas of degraded, xerothermic scrub, parasitizing Artemisia spp. (A. barrelieri Besser, A. campestris L. subsp. glutinosa [J. Gay ex DG.] Batt., A. caerulescens L. subsp. gallica [Willd.] K. M. Perss., A. herba-alba Asso) and Launaea lanifera Pau, at altitudes between sea level and $1500 \mathrm{~m}$. Anthesis was from March to June. The main distribution areas for this species in the Iberian Peninsula are the north-east, east, and south-east but it is also found in the central region (Fig. 3).

Orobanche cumana was exclusively found on cultivated land, as a parasite of sunflower (Helianthus annuus L.), on alluvial loamy soils at altitudes between sea level and $800 \mathrm{~m}$. Anthesis was from June to August. It is distributed throughout the area of cultivation of sunflower in the central and southern Iberian Peninsula (Fig. 3).

The above results indicate that $O$. cernua and $O$. cumana have a different distribution within the Iberian Peninsula, where they are adapted to different ecological niches. They do not coexist but their distribution areas are nearly in contact in central and southern Spain. The possibility of cross-pollination is very low due to differences in anthesis period. No populations of $O$. cermua have been detected on cultivated sunflower nor have plants of $O$. cumana been reported parasitizing wild species in the Iberian Peninsula, despite the fact that both $O$. cumana was introduced and sunflower cultivation commenced in Spain some decades ago (Díaz-Celayeta, 1974).

\section{Oil content and fatty acid composition}

The analysed populations of O. cernua and O. cumana were characterized by similar levels of seed oil content, but clearly differed in their fatty acid composition (Table 3). Oleic acid was the predominant fatty acid in the seed oil of all populations of O. cernua, ranging from $49.6 \%$ to $68.1 \%$, whereas linoleic acid was predominant in 


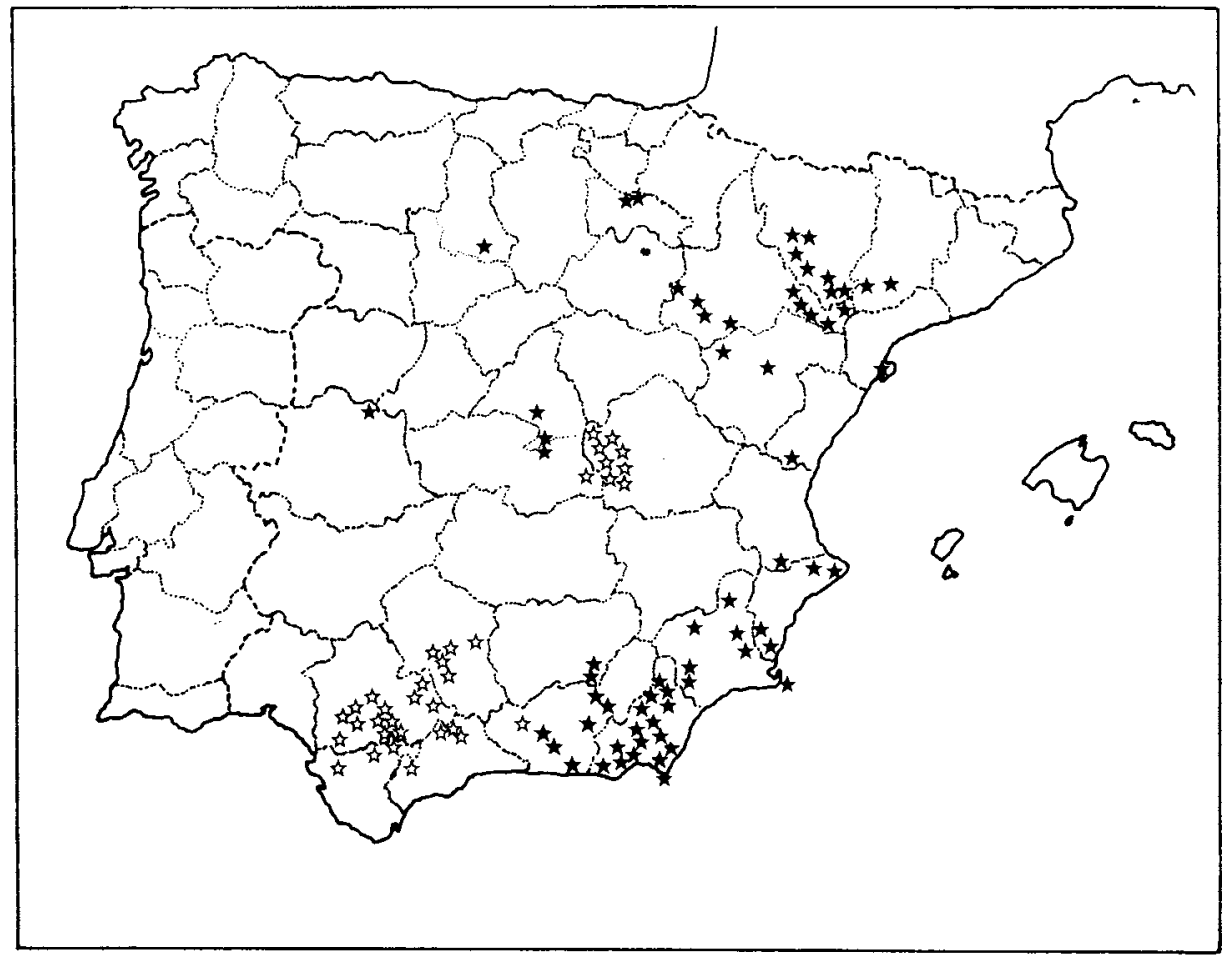

Figure 3. Distribution of Orobanche cernua $(\star)$ and O. cumana (§)).

TABLE 3. Number of analysed populations, seed oil content ( $\%$ fresh seed weight), and fatty acid composition (\% of the total fatty acids) of Orobanche cernua and O. cumana from the Iberian Peninsula

\begin{tabular}{|c|c|c|c|c|c|c|c|c|}
\hline \multirow[b]{2}{*}{ Trait } & \multicolumn{4}{|c|}{ O. cernua } & \multicolumn{4}{|c|}{ O. cumana } \\
\hline & $n$ & Mean & Min. & Max. & $n$ & Mean & Min. & Max. \\
\hline Oil & 27 & 20.2 & 10.7 & 32.1 & 17 & 18.3 & 11.1 & 29.1 \\
\hline Palmitic acid & 27 & 5.0 & 3.4 & 6.9 & 17 & 2.9 & 1.7 & 4.5 \\
\hline Stearic acid & 27 & 1.9 & 1.0 & 4.2 & 17 & 1.3 & 1.0 & 1.9 \\
\hline Oleic acid & 27 & 57.9 & 49.6 & 68.1 & 17 & 34.8 & 29.7 & 43.4 \\
\hline Linoleic acid & 27 & 35.2 & 26.1 & 39.6 & 17 & 61.0 & 53.0 & 66.3 \\
\hline
\end{tabular}

O. cumana, with concentrations from $53.0 \%$ to $66.3 \%$ (Table 3). Both species also differed in their concentrations of the saturated palmitic and stearic acid, which were present at higher concentrations in O. cernua. Figure 4 shows the frequency distribution for linoleic acid concentration in the analysed populations, which clearly reveals the differences for this trait between both species. There are previous studies revealing that quantitative differences in the seed oil fatty acid proflile may act as chemotaxonomic markers, for example in the genera Linum, Linaceae (Rogers, 1972) and Epilobium, Onagraceae (Velasco \& Goffman, 1999). Although the significance and potential contribution of this trait to the infrageneric classification of Orobanche has not been yet defined, the consistent differences for seed oil fatty acids between 


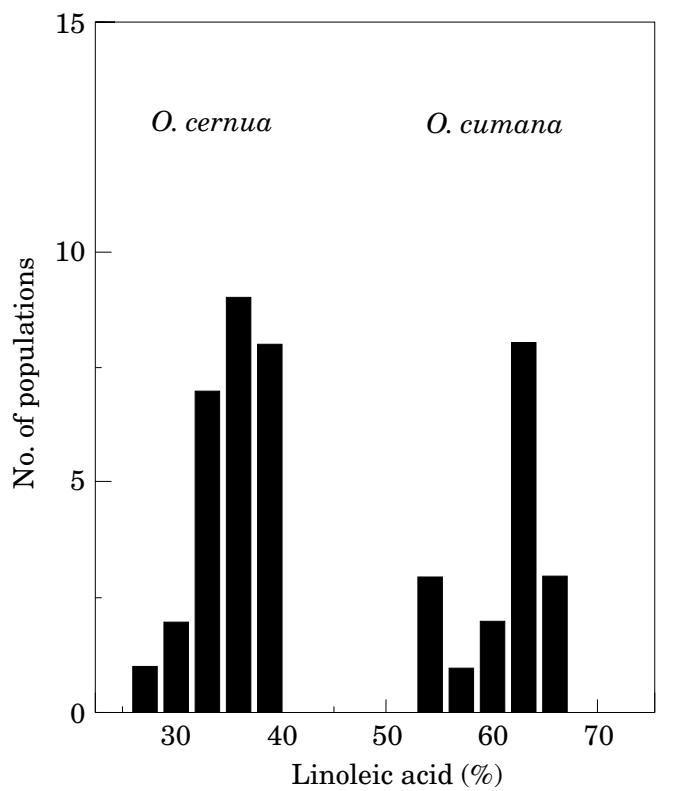

Figure 4. Frequency distribution of linoleic acid concentration ( $\%$ of the total seed oil fatty acids) in 27 populations of Orobanche cernua and 17 populations of O. cumana.

O. cernua and O. cumana populations in the Iberian Peninsula might be relevant for the classification of both taxa.

\section{CONCLUSIONS}

Our evaluation of O. cernua and O. cumana populations in the Iberian Peninsula revealed significant differences between both species. Orobanche cumana is an allochthonous species of the Iberian Peninsula, with its main distribution area in central Asia and eastern Europe. In contrast, O. cernua is an autochthonous species mainly distributed in the Mediterranean basin and in markedly arid areas eastwards. The results of the present study revealed that the populations of both taxa in the Iberian Peninsula show clear ecological, morphological and biochemical differences, thus giving additional support to those authors considering them as separate species.

\section{ACKNOWLEDGEMENTS}

The authors thank DrJ. M. Melero-Vara and Prof. DrJ. M. Fernández-Martínez, Instituto de Agricultura Sostenible (CSIC) at Córdoba, Spain, for providing valuable populations of $O$. cumana for seed oil characterization, and to the keepers of the Herbaria ABH, ALME, BC, BCG, BCF, $\mathrm{C}^{\circ}$ LA SALLE DE ALMERIA, GDA, GDAC, JACA, JAEN, HBIL, MA, MACB, MAF, MGC, MUB, SEV, VAB \& VAL for the loan of the material. 


\section{REFERENCES}

Abu-Irmaileh BE. 1994. Overview of the Orobanche problem in the Near East. In: Pieterse AH, Verkleij JAC, Ter-Borg SJ, eds. Proceedings of the Third International Workshop on Orobanche and Related Striga Research. Amsterdam, 677-683.

Aitzetmüller K, Tsevegsüren N, Werner G. 1999. Seed oil fatty acid patterns of the AconitumDelphinium-Helleborus complex. Plant Systematics and Evolution 215: 37-47.

Alonso LG, Fernández-Escobar J, López G, Rodríguez-Ojeda MI, Sallago F. 1996. New highly virulent sunflower broomrape (Orobanche cernua Loefl.) pathotypes in Spain. In: Moreno MT, Cubero JI, Berner D, Joel D, Musselman LJ, Parker C, eds. Proceedings of the Sixth International Parasitic Weed Symposium. Córdoba, 639-644.

Antonova TS, Ter-Borg SJ. 1996. The role of peroxidase in the resistance of sunflower against Orobanche cumana in Russia. Weed Research 36: 113-121.

Beck-Mannagetta G. 1890. Monographie der Gattung Orobanche. Bibliotheca Botanica 19: 1-275.

Beck-Mannagetta G. 1930. Orobanchaceae. In: Engler A, ed. Das Pfanzenreich, vol. IV. Leipzig: Verlag von Wilhelm Engelmann, 1-348.

Bischof F. 1978. Common Weeds from Iran, Turkey, the Near East and North Africa. Eschborn: Deutsche Gesellschaft für technische Zusammenarbeit (GTZ).

Bonnier G. 1926. Flore complète illustrée en couleurs de France, Suisse et Belgique. Tome VIII, fasc. 78. Paris: Librairie Générale de L'Enseignement.

Gastejón-Muñoz M, Romero-Muñoz F, García-Torres L. 1989. Áreas de distribución del jopo de girasol en Andalucía. Agricultura 688: 981-982.

Gastejón-Muñoz M, Romero-Muñoz F, García-Torres L. 1991. Orobanche cernua dispersion and its incidence in sunflower in Andalusia (Southern Spain). In: Wegmann K, Musselman LJ, eds. Proceedings of the International Workshop on Orobanche Research. Tübingen: Eberhard-Karls University, $44-48$.

Chater AO, Webb DA. 1972. Orobanchaceae. In: Tutin TG, Heywood VH, Burges NA, Moore DM, Valentine DH, Walters SM, Webb DA, eds. Flora Europaea, vol. 3. Cambridge: Cambridge University Press, 285-294.

Gherzhentseava MI. 1978. Inheritance of broomrape (Orobanche cumana) resistance in sunflower: control of the parasite. Soviet Genetics 14: 1254-1256.

Coste H. 1937. Flore descriptive et illustrée de la France, de la Corse et des contrées limitrophes. Vol III. Paris: Librairie Scientifique et Technique Albert Blanchard.

Díaz-Celayeta F. 1974. Algunas plantas parásitas de otras de interés agrícola o medicinal. Anales INIA Serie Protección Vegetal 4: 143-160.

Encheneva V, Shindrova P. 1994. Broomrape (Orobanche cumana Wall.) - a hindrance to sunflower production in Bulgaria. In: Pieterse AH, Verkleij JAC, Ter-borg SJ, eds. Biology and management of Orobanche: Proceedings of the Third International Workshop on Orobanche and related Striga research. Amsterdam, 619-622.

Garcés R, Mancha M. 1993. One-step lipid extraction and fatty acid methyl esters preparation from fresh plant tissues. Analytical Biochemistry 211: 139-143.

Gibbs RD. 1974. Chemotaxonomy of flowering plants. Vol. I. Constituents. London: McGill-Queen's University Press.

González-Torres R, Jiménez-Díaz RM, Melero-Vara JM. 1982. Distribution and virulence of Orobanche cernua in sunflower crops in Spain. Phytopathologische Zeitschrift 104: 78-89.

Greuter W. 1988. International code of botanical nomenclature. Königstein: Koeltz Scientific Books.

Greuter WR, Burdet HM, Long G. 1989. Med-checklist. Vol. 4 (Lauraceae-Rhamnaceae). Genève: Conservatoire et Jardin Botaniques de la Ville de Genève.

Jackson BD. 1895. Index Kewensis. Vol. 2. Oxford: Clarendon Press, 372.

Joel DM. 1987. Identification of Orobanche seeds. In: Weber HC, Forstreuter W, eds. Parasitic Flowering Plants, Proceedings of the 4th ISPFP. Marburg, 445-448.

Joel DM. 1988. Orobanche cumana: a new adventive weed in Israel. Phytoparasitica 16: 375.

Joel DM, Portnoy VH, Katzir N. 1998. Use of DNA fingerprinting for soil-borne seed identification. Aspects of Applied Biology 51: 23-27.

Katzir N, Portnoy V, Tzuri G, Gastejón-Muñoz M, Joel DM. 1996. Use of random amplified polymorphic DNA (RAPD) markers in the study of the parasitic weed Orobanche. Theoretical and Applied Genetics 93: 367-372. 
Kreutz CAJ. 1995. Orobanche: the European Broomrape Species. I. Central and Northern Europe. Maastricht: Stichting Natuurpublicaties Limburg.

Loefling P. 1758. Iter Hispanicum, eller resa til Spanska länderna uti Europa och America. Stockholm: Salvius.

López González G. 1990. La obra botánica de Löfling en España. In: Pelayo F, ed. Pehr Löfling y la Expedición al Orinoco, 1754-1761. Madrid: Real Jardín Botánico, 33-49.

Paran I, Gidoni D, Jacobsohn R. 1997. Variation between and within broomrape (Orobanche) species revealed by RAPD markers. Heredity 78: 68-74.

Parker C. 1994. The present state of the Orobanche problem. In: Pieterse AH, VerkleijJAC, Ter-borg SJ, eds. Biology and management of Orobanche: Proceedings of the Third International Workshop on Orobanche and related Striga research. Amsterdam, 17-26.

Petrov D. 1968. A new physiological race of broomrape in Bulgaria (Orobanche cumana Wallr.). Comptes Rendus de l'Academie des Sciences Agricoles en Bulgarie 1: 27-30.

Pignatti S. 1982. Flora d'Italia. Vol. II. Bologna: Edagricole.

Pujadas A, Thalouarn P. 1998. O. cernua Loefl. \& O. cumana Wallr. in the Iberian Peninsula. Proceedings of the 6th Mediterranean Symposium EWRS. Montpellier, 159-160.

Rechinger KH. 1943. Flora Aegea: Flora der Insel und Halbinseln des Aegaeischen Meeres. Wien: Springer.

Reichenbach HGL. 1829. Iconographia botanica seu plantae criticae. Icones rariorum et minus rite cognitarum. Vol. VII. Lipsiae: Hofmeister.

Reuter GF. 1847. Orobanchaceae. In: De Candolle AP, ed. Prodromus systematis naturalis regni vegetabilis 11: 1-45. Paris.

Rogers CM. 1972. The taxonomic significance of the fatty acid content of seeds of Linum. Brittonia 24: $415-419$

Sukno S, Melero-Vara JM, Fernández-Martínez JM. 1999. Inheritance of resistance to Orobanche cernua Loefl. in six sunflower lines. Crop Science 39: 674-678.

Uhlich H, Pusch J, Barthel KJ. 1995. Die Sommerwurzarten Europas. Magdeburg: Westarp Wissenschaften.

Velasco L, Goffman FD. 1999. Tocopherol and fatty acid composition of twenty-five species of Onagraceae Juss. Botanical fournal of the Linnean Society 129: 359-366.

Velayos-Rodríguez M. 1978. Aportación al estudio de las malas hierbas de los cultivos de girasol. Unpublished Thesis, Universidad Complutense, Madrid.

Venkov V, Bozoukov H. 1994. Influence of different species of Orobanche on cultivated sunflower and tobacco. In: Pieterse AH, Verkleij JAC, Ter-borg SJ, eds. Biology and management of Orobanche: Proceedings of the Third International Workshop on Orobanche and related Striga research. Amsterdam, 349-350.

Vrânceanu AV, Pirvu N, Stoenescu FM, Pacureanu M. 1986. Some aspects of the interaction Helianthus annuus L./ Orobanche cumana Wallr. and its implications in sunflower breeding. In: Pieterse AH, Verkleij JAC, Ter-borg SJ, eds. Biology and management of Orobanche: Proceedings of the Third International Workshop on Orobanche and related Striga research. Amsterdam, 181-189.

Wallroth KFW. 1825. Orobanches Generis. Francofurti ad Moenum: Frederic Wilmans. 